https://doi.org/10.48009/2_iis_2005_83-89

\title{
PRIVACY IN E-COMMERCE: UNDERSTANDING USER TRADE-OFFS
}

\author{
Dr. Silvana Faja, Central Missouri State University, sfaja@cmsu1.cmsu.edu
}

\begin{abstract}
Concern about the privacy of personal information is one of the issues associated with ecommerce. This paper focuses on privacy concerns in the context of online environment, to better understand its nature and identify the trade-offs Internet users are willing to make when disclosing personal information to web sites. The effect that benefits such as compensation and personalization have on privacy concerns are analyzed through a survey of potential users.
\end{abstract}

Keywords: e-commerce, privacy concern, Internet-user attitudes, personalization

\section{INTRODUCTION}

Since the 1980s, with advances in information technology, there has been an increasing trend in the concern about information privacy [9]. This has made issues related to privacy relevant research topics in several fields. Information privacy has been viewed from philosophical, legal, business, and technical perspectives because, as Ackerman et al. [1] put it, privacy forms a codesign space between the social, technical, and regulatory. In the area of information systems, information privacy has been considered as the most important ethical issue of the information age and as one of the key information liability issues facing managers [12, 21]. In fact, gaining strategic advantage through information technology frequently means relying on the secondary use of the information collected [4]. Fear of loss of personal privacy associated with the emerging e-marketplace is one of the key issues identified by Internet users. As the technology to collect, store and process data is advancing, there is a growing concern among them about the privacy of information collected online [3, 14].

Research suggests that privacy concerns are highly contextual $[17,18]$ and that is important to understand the thresholds of privacy concerns that are manifested in the users' behavior online. For instance, despite their stated concern about privacy, online customers are generally quick to provide significant amount of personal information when given an incentive [22]. This paper focuses on online privacy concern aiming at understanding the trade-off between users' privacy concerns and benefits from disclosing personal information while interacting with e-commerce web sites. The paper tries to answer the following questions: 1) Does the type of benefit received in exchange for information affects users privacy concerns? and 2) Do people with different levels of privacy concerns perceive these benefits differently?

\section{THEORETICAL BACKGROUND}

Privacy has been described as "the right to be left alone" [6]. In the context of activities that take place in the electronic marketplace privacy refers to personal information, while invasion of privacy usually means the unauthorized collection, disclosure or other uses of personal information as a direct result of online transactions [23]. 
Previous research has attempted to identify dimensions of privacy concerns. Smith et al. [20] identified four dimensions of privacy concern that are Awareness of Data Collection, Secondary Use, Access, and Errors. The first two factors are generally considered the most important aspects of privacy concern. Awareness of Data Collection is defined as a general concern that an extensive amount of identifiable information is being collected and stored in databases.

Secondary Usage refers to the concern that information that is collected from individuals for one purpose is used for another, secondary purpose, without authorization from the individuals. Smith et al. [20] distinguished two types of such usage: internal and external usage. Internal secondary usage refers to the use of information within a single organization. External usage refers to the disclosure of information to external parties or other organizations. Sheehan and Hoy [20] identified three factors that influence privacy concerns: control over collection and usage of information, short term transaction, and established relationship. Culnan [4] explored consumer attitudes toward secondary use of information. Their study found that people who are less sensitive about secondary use of information have a lower concern for privacy. A study by Dhillon and Moores [5] revealed that the main privacy issue for Internet users was that companies should not sell personal information. Cranor et al. [3], identified as the most important factor whether or not information would be shared with other organizations. Improper transfer, which includes the transfer of a consumer's information to other businesses without notice or the acknowledgement from the customer, was also identified as one of the aspects of privacy concern by Wang et al. [23].

Along with privacy concerns, research has also identified the conflicts that exist between privacy and other interests. One such conflict is that between two or more consumer demands [6]. For instance, in order to receive the desired level of service consumers may be willing to disclose personal information. Analysis of these aspects has been based by some researchers on the social contract theory. A social contract emphasizes the role of norms of behavior [13]. Kaufman et al. [10] described a social contract as 'defining the collective rules that constraint the behavior of individuals and groups living in a society in such a way as to protect the individual, while also benefiting the society as a whole.' In the context of technology, social contracts emerge as accepted conventions between people that evolve as they adapt to new technologies. Research in sociology shows that individuals decide to disclose personal information in an exchange for some benefits based on an assessment of how their personal information will be subsequently used and whether they will suffer negative consequences in the future [11]. Individuals will engage in social contracts as long as benefits exceed risks $[13,5]$.

Past research has analyzed privacy concerns in the context of benefits received in exchange for disclosing information such as financial rewards, access to information of interest, or other offers $[13,15,19,2]$. For instance, Hann et al., [7] conducted a study that attempted to measure the dollar value of information privacy concern. In that study, the economic benefit was measured along two dimensions: monetary reward and future time savings. They found that individuals are willing to trade off privacy concern for economic benefits. Based on these arguments, the following was hypothesized:

Hypothesis 1: An individual's concern when disclosing information in exchange for benefits will be lower compared to general concern about collection and usage of personal information. 
Research suggests that people often consider the nature of benefit they receive in exchange for information when they decide whether an activity violates their personal privacy [24]. To explore this proposition, this study explored the following hypotheses:

Hypothesis 2: The type of benefit received in exchange for personal information affects user's concern about privacy.

Two types of benefits were considered in this study: compensation and personalization. Personalization allows companies to make suggestions to customers from previous purchases and profiles and also transforms a standard product or service to a specialized solution for an individual. A survey conducted by Privacy \& American Business, demonstrated that a significant majority of Internet users desire information that is tailored to their needs and are willing to provide their buying preferences in order to receive personalized ads [24]. More than two-thirds of Internet users $(68 \%)$ in that survey said they would provide personal information in order to receive tailored banner ads.

Research has shown that individuals differ in their propensity to be concerned about information privacy $[1,18]$. These studies have introduced typologies to categorize Internet users based on their general attitude about privacy. It is suggested that different categories, may perceive privacy related aspects differently. For instance, Jaisingh et al. [8] conducted an economic analysis of the value of information privacy focusing on Internet Service Providers (ISP). They found that an ISP or any firm that can collect information should have separate strategies for high privacy and low privacy types of customers. Thus, the following hypothesis is considered:

Hypothesis 3: The level of control over personal information moderates the effect of type of benefit on privacy concern.

In addition, the following hypotheses were tested in this study: Hypothesis 4: Past purchasing behavior affects an individual's concern about the collection and usage of personal information.

Hypothesis 5: Past purchasing behavior affects an individual's concern about disclosing information in exchange for benefits.

\section{METHODOLOGY}

These hypotheses were tested using a survey. Items used to measure the constructs were adopted from previous research. Privacy concern was operationalized as the control over the collection and usage of information and its measure was adopted from Sheehan and Hoy [19]. This measure consists of five items representing five scenarios. Four items measured awareness of data collection and two items measured concern about secondary use of information, both internally and externally to other companies. The scale to measure compensation was also adapted from Sheehan and Hoy. Personalization scale was adopted from Cranor et al. [3]. The level of concern of the respondents in each of the scenarios was measured in a seven point bipolar scale where 1 represented the lack of any concern at all and 7 represented extreme concern. 
Before hypotheses testing, validity and reliability analyses were performed using SPSS statistical package. An exploratory principal components factor analysis was used to examine the extent to which various constructs measured in the study showed discriminant validity. One item showed cross-loading and was dropped from further analyses. The reliability analysis showed a good internal consistency with Cronbach's Alpha ranging from 0.79- 0.84.

Data were collected from students enrolled in business courses at a Midwestern University. The total number of subjects that participated in the study was 104 . The number of valid responses was 101 . The sample consisted of 57 males and 43 females and $91 \%$ were in the age group 1824. Sixty-six percent of the respondents had purchased online in the past year and $72 \%$ were registered with a web site.

\section{ANALYSIS AND RESULTS}

Initially subjects were divided into groups according to their level of privacy concern. This grouping was needed for the testing of Hypothesis 3 that considers the moderating role of level of privacy concern. The grouping of subjects was achieved using cluster analysis, specifically the TwoStep Cluster Analysis procedure in SPSS. This clustering technique was chosen because the procedure can automatically determine the optimal number of clusters, instead of the researcher specifying the number of clusters. This analysis revealed two groups of subjects based on their privacy concern. Based on the group (cluster) means, subjects classified in the first group (mean $=3.03$ ) were considered with a lower level of privacy concern and those of the second group $($ mean $=5.32)$ with a higher level of privacy concern.

To test Hypotheses 1 and 2 paired samples t-tests were conducted using SPSS to explore the differences between the three different concerns. Results of these analyses are shown in Table 1. These tests showed that concern when information is disclosed in exchange for benefits was significantly lower than the general concern about the control over collection and usage of data. Thus, Hypothesis 1 was supported. To further explore these differences, the same tests were conducted for each cluster of subjects. For the first cluster, concern over data disclosed for personalization was significantly higher than the general concern $(\mathrm{t}(34)=-3.28, \mathrm{p}<.05)$. However, concern over data disclosed for compensation did not differ significantly from the general concern about control of information $(\mathrm{t}(34)=1.85, \mathrm{p}>.05)$. For the second cluster, concern over data disclosed for personalization was significantly lower than general concern $(\mathrm{t}(65)=6.59, \mathrm{p}<.01)$. Also, concern over data disclosed for compensation was significantly lower than general concern about control of data $(\mathrm{t}(65)=7.28, \mathrm{p}<.01)$. The $\mathrm{t}$-test for Hypothesis 2 showed that there was no significant difference in the privacy concern when information is disclosed in exchange for compensation versus personalization, which leads to rejection of Hypothesis 2.

Table 1. Results of t-tests for the differences in concerns

\begin{tabular}{lcccc} 
Privacy concern & $\begin{array}{c}\text { Mean } \\
\text { difference }\end{array}$ & $\mathbf{t}$ & df & Sig \\
\hline Control vs. compensation & 0.74 & 6.856 & 100 & $0.000^{*}$ \\
Control vs. personalization & 0.47 & 3.444 & 100 & $0.001^{*}$ \\
Compensation vs. personalization & 0.05 & 0.374 & 100 & 0.709
\end{tabular}


In order to test Hypothesis 3, a 2 X 2 MANOVA (clusters of subjects by type of benefit) for repeated measures was carried out. Results of this analysis are presented in Table 2 . This analysis yielded a significant main effect of clusters of subjects, $F(1,99)=31.27$, p $<.001$ as well as a significant interaction effect between the type of benefit and clusters of subjects, F (1, $99)=7.60, \mathrm{p}<.01$.

Table 2. Results of MANOVA

\begin{tabular}{lllll} 
Source & df & Mean Square & F & Sig. \\
\hline Benefit & 1 & .178 & 0.229 & .633 \\
Clusters & 1 & 69.932 & 31.27 & $.000^{*}$ \\
Benefit*Cluster & 1 & 5.898 & 7.602 & $.007^{*}$
\end{tabular}

A follow up analysis was conducted to examine which particular groups differed. Pairwise comparisons using the Bonferroni procedure showed that subjects of the first cluster (lower privacy concern) had a significantly lower concern for both types of benefits (mean $=2.95$ ) compared to those of the second cluster $($ mean $=4.19)$. In addition, paired samples t-tests were conducted for each cluster. These tests revealed that for subjects with lower concern about the collection and usage of data (cluster 1), there were no significant differences between the concern about the information disclosed in the context of personalization versus compensation $(\mathrm{t}(34)=-1.864, \mathrm{p}>.05)$. For subjects in cluster 2 , concern about disclosing information in exchange for compensation was significantly higher than concern about disclosing information in exchange for personalization $(\mathrm{t}(65)=2.018, \mathrm{p}<.05)$. Thus, Hypothesis 3 was supported. An individual's level of control over personal information moderates the effect of type of benefit on privacy concern.

To test $\mathrm{H} 4$ and $\mathrm{H} 5$ on the role of past purchasing behavior, three regression analyses were conducted. The independent variable in these analyses was previous online purchases. The dependent variables were general concern on privacy for the first regression analysis, concern about disclosing information in exchange for personalization in the second analysis, and concern about disclosing information in exchange for compensation in the third one. Results showed that none of the relationships were significant. For the first regression $\beta=0.94, t=.944, p>.05$, for the second regression $\beta=0.05, \mathrm{t}=0.502, \mathrm{p}>.05$ and for the third one $\beta=0.63, \mathrm{t}=0.625, \mathrm{p}>.05$. None of these hypotheses were supported.

\section{DISCUSSION}

Internet holds a tremendous potential for businesses and consumers, but its use also arises a lot of concern about privacy protection. This paper tried to explore privacy concerns among online users and how these concerns vary based on context. Previous research has suggested that people are willing to disclose information in exchange for benefits. These benefits can be in the form of financial compensation, or ability to access information of interest, or can be in the form of personalized service or products. This proposition was supported in this study. Results suggested that concern about disclosing information in exchange for benefits were lower compared to the general concern about collection and usage of personal data. Interestingly, this study showed that the effect of benefit was not the same for all subjects. While the above proposition was true for subjects with relatively high level of concern, subjects with lower general concern about control 
were more concerned about disclosing data in exchange for personalization. This is an indication of the fact that in spite of general attitude, concern about information disclosed depends on the situation.

The study explored possible differences between users' perceptions of different forms of benefits. It was hypothesized that the type of benefit may influence concern about disclosure. The study showed that in general, the concern about disclosing information in exchange for some kind of material compensation did not differ significantly from the concern when information is disclosed to receive personalized service or products. However, it was suggested that this relationship is influenced by individual's general concern about collection and usage of information online. While there were no differences between the two forms of benefits for individuals with relatively lower general concern, differences were observed for subjects with higher levels of concern. They were more concerned to disclose information for compensation than personalization. This finding is similar to that found in another study where reward actually heightened subjects' concerns [2]. Finally, the study showed that previous purchasing behavior did not affect any of the privacy concerns considered in this study.

This study was exploratory in nature. Although past research has considered factors such as financial reward in exchange for information, little has been done to compare users' perceptions about different types of benefits. Personalization is a factor that has not been considered by prior research. Personalization is important for web sites because it creates a user experience that is both compelling and sticky [17]. As such, e-vendors may want to understand what forms of compensation or benefits are most valued by their targeted audiences. From the practice perspective, a better understanding of these relationships will lead to better policy decisions and better design of web sites.

In addition to contributions, this study has also some limitations. One limitation may be the lack of generalizability, because of the homogeneity of the sample. It cannot be assumed that the findings can be extended for any type of online users, especially with regard to age and education.

\section{REFERENCES}

1. Ackerman, M., Darrell, T. \& Weitzner, D.J. (2001). Privacy in context. Human-Computer Interaction, 16, pp.167-176.

2. Andrade, E.B., Kaltcheva, V. \& Weitz, B. (2002). Self-disclosure on the Web: The impact of privacy policy, reward, and company reputation. Advances in Consumer Research, 29, pp.350-353.

3. Cranor, L. F., Reagle, J. \& Ackerman, M. S. (1999). Beyond concern: Understanding Net Users's Attitudes About Online Privacy, AT\&T Labs-Research Technical Report TR 99.4.3, [available at http://www.research.att.com/library/] (accessed October 30, 2001).

4. Culnan, M. J. (1993). How did they get my name?: An exploratory investigation of consumer attitudes toward secondary information use. MIS Quarterly, 17, pp. 341-361.

5. Dhillon, G.S. \& Moores, T.T. (2001). Internet privacy: Interpreting key issues. Information Resources Management Journal, 14(4), pp.33-37. 
6. Goodwin, C. (1991). Privacy: Recognition of a Consumer Right, Journal of Public Policy \& Marketing, 10(1), pp.149-166.

7. Hann, I., Hui, K.L., Lee, T.S. \& Png, I.P.L. (2002). Online information privacy: Measuring the cost-benefit trade-offs. Proceedings of the Twenty-Third International Conference on Information Systems, Barcelona, Spain, pp.1-10.

8. Jaisingh, J., Barron, J., Chaturvedi, A. \& Mehta, S. (2002). Privacy on the Internet: An economic analysis. Proceedings of the Eighth Americas Conference on Information Systems, Dallas, Texas, USA, pp. 361-374.

9. Katz, J.E. \& Tassone, A.R. (1990). A report: Public opinion trends: Privacy and information technology. Public Opinion Quarterly, 54 (1), pp.125-143.

10. Kaufman, J.H., Edlund, S., Ford, D.A. \& Powers, C. (2002). The social contract core. Proceeding of the $11^{\text {th }}$ International Conference on World Wide Web, Honolulu, Hawaii, USA, pp.210-220.

11. Laufer, R.S. \& Wolfe, M. (1977). Privacy as a concept and a social issue: A multidimensional developmental theory. Journal of Social Issues, 33, pp.22-42.

12. Mason, R.O. (1986). Four ethical issues of the information age. MIS Quarterly, 10(1), pp.412.

13. Milne, G.R. \& Gordon, M.E. (1993) Direct mail privacy-efficiency trade-offs with an implied social contract framework. Journal of Public Policy and Marketing, 12(2), pp. 206215.

14. O'Neil, D. (2001). Analysis of Internet users' level of online privacy concerns. Social Science Computer Review, 19 (1), pp. 17-31.

15. Phelps, J. E; Nowak, G. J. \& Ferrell, E. (2000). Privacy concerns and consumer willingness to provide personal information. Journal of Public Policy and Marketing, 19 (1), pp.27-41.

16. Riedl, J. (2001). Personalization and Privacy. IEEE Internet Computing, 5(6), pp.29-31.

17. Schoeman, F.D. (1984). Philosophical dimensions of privacy: an anthology. Cambridge, NY: Cambridge University Press.

18. Sheehan, K.B. (2002). Toward a typology of Internet users and online privacy concerns. The Information Society, 18, pp. 21-32.

19. Sheehan, K. B. \& Hoy, M. G. (2000). Dimensions of Privacy Concern Among Online Customers, Journal of Public Policy \& Marketing, 19(1), pp. 62-73.

20. Smith, H. J., Milberg, S. J. \& Burke, S, J. (1996). Information privacy: Measuring individuals' concerns about organizational practices," MIS Quarterly, 20(2), pp.167-195.

21. Straub, D.W. \& Collins, R.W. (1990). Key information liability issues facing managers: Software piracy, proprietary databases, and individual rights to privacy. MIS Quarterly, 14 (2), pp. 143- 157.

22. Tweney, D. (1998). The consumer battle over online information has just begun, Infoworld, 20(25), pp.66

23. Wang, H., Lee, M.K.O. \& Wang C. (1998). Consumer Privacy Concerns about Internet Marketing, Communications of the ACM, 41 (3), pp.63-70.

24. Westin, A. (1997). Privacy and American Business Study, [available at http://www.pandab.org] (accessed October 29, 2001) 